\section{Check for updates}

Cite this: Mater. Adv., 2021, 2, 3736

Received 26th March 2021, Accepted 14th April 2021

DOI: $10.1039 / \mathrm{d} 1 \mathrm{ma} 00260 \mathrm{k}$

rsc.li/materials-advances

\title{
Polymeric near infrared emitters with bay-annulated indigo moieties $\dagger$
}

\author{
Ana Clara B. Rodrigues, (D) ${ }^{a}$ Anika Eckert, ${ }^{b}$ João Pina, (D) ${ }^{a}$ Ullrich Scherf (D) ${ }^{b}$ and \\ J. Sérgio Seixas de Melo (D)*a
}

\begin{abstract}
Three new copolymers based on bay-annulated indigo (BAI) building blocks were synthesized through Stille-type cross-coupling reactions, alternating the electron-deficient BAI units with thiophene-based donor moieties. These polymers showed absorption and fluorescence emission in the NIR region. Their spatial structures were found to prevent aggregation-caused quenching (ACQ) in the solid state. Photoluminescence at $77 \mathrm{~K}$ is found $\mathrm{ca}$. four times more intense than at $293 \mathrm{~K}$. Based on time-resolved photoluminescence experiments, the excited-state energy transfer hopping deactivation in the copolymers is discussed in terms of a Förster-type mechanism, with time constants ranging from $10^{10}$ to $10^{11} \mathrm{~s}^{-1}$.
\end{abstract}

\section{Introduction}

Natural dyes and pigments as indigo (Scheme 1) and its natural (indigoids) or synthetic derivatives are valued for their bright color and high photochemical stability and have been used since antiquity. ${ }^{1-5}$ A bay-annulated indigo derivative known as Cibalackrot (7,14-diphenyldiindolo[3,2,1-de;3 $\left.3^{\prime}, 2^{\prime}, 1^{\prime}-i j\right][1,5]$ naphthyridine-6,13-dione), an industrial dye that has been known for over a century, ${ }^{6}$ has a highly rigid chemical structure, showing strong luminescence ${ }^{7}$ and is, as indigo, capable of singlet fission $^{8,9}$ and can be used as an organic laser dye. ${ }^{10}$

The need for better performing and stable organic materials in organic electronics inspired the resurgence of historically long known molecules as indigo ${ }^{11,12}$ and their subsequent chemical transformation into new families of $\pi$-conjugated building blocks, ${ }^{12}$ also for construction of new macromolecular semiconductors. ${ }^{11}$ In this work, indigo was functionalized by reaction with 2-thienylacetyl chloride, as described previously, ${ }^{13,14}$ to produce the bay-annulated indigo derivative BAI1 (Scheme 1). The electron-deficient BAI1 moiety is a suitable building block for the generation of push-pull type conjugated polymers. ${ }^{15}$ In recent years copolymers comprising these building blocks have been studied for their suitability in OFETs and OPVs applications. ${ }^{13}$ Moreover, these donor-acceptor copolymers are also attractive in

\footnotetext{
${ }^{a}$ University of Coimbra, CQC, Department of Chemistry, P3004-535 Coimbra, Portugal.E-mail: sseixas@ci.uc.pt

${ }^{b}$ Bergische Universität Wuppertal, Macromolecular Chemistry Group (buwmakro) and Wuppertal Center for Smart Materials and Systems (cm@s), Gauss-Str.20, D-42119, Wuppertal, Germany

$\dagger$ Electronic supplementary information (ESI) available: For detailed synthesis, structure characterization and further photophysical data. See DOI: 10.1039/ d1ma00260k
}

view of a potential application in organic solar cells ${ }^{16}$ and as NIR detectors. More specifically, near-infrared photodetection (NIR-OPD, (a)800-3000 nm $)^{17}$ is valuable for numerous scientific as well as industrial (inspection, sorting, safety/security) and recreational applications. Nowadays, near-infrared photodetectors are commonly based on inorganic semiconductors such as InGaAs, InGaSb ${ }^{18}$ or HgCdTe, ${ }^{19}$ although organic-based NIR detectors ${ }^{18,20-24}$ have gained increasing interest. To date, a very limited amount of well performing NIR-OPDs have been reported. ${ }^{24}$ Recently, push-pull copolymers based on BAI indigo core were found to be suitable for photodetection beyond $1000 \mathrm{~nm}^{19}$

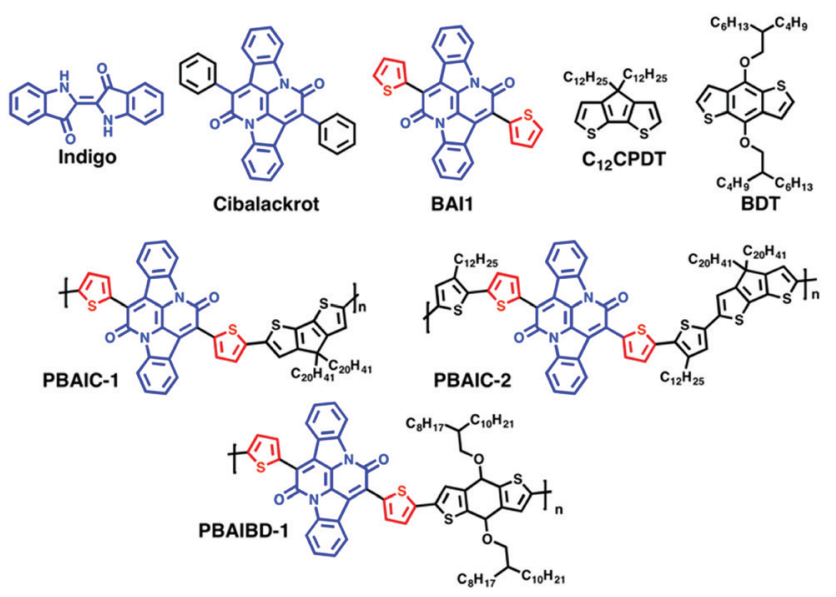

Scheme 1 Structures and acronyms of indigo and its bay-annulated derivates, Cibalackrot and BAI1, together with the chemical structures of the thiophene-based building blocks ( $\mathbf{C}_{12}$ CPDT and BDT) used to prepare the NIR-emitting copolymers: PBAIC-1, PBAIC-2 and PBAIBD-1. 
Here, we investigate the ground- and excited-state characteristics of copolymers comprising BAI1 units, linked through thiophene-based donor units (cyclopentadithiophene CPDT- or benzodithiophene BDT-based) to verify the potential of such copolymers as NIR solid state emitters. Rational design considerations of the polymers indicate that the introduction of planarized and symmetrical thiophene donors (CPDT, $4 H$ cyclopenta[1,2- $\left.b: 5,4-b^{\prime}\right]$ dithiophene, and BDT, benzo[1,2- $b: 4$, $\left.5-b^{\prime}\right]$ dithiophene) should enable interchain $\pi-\pi$ stacking and maximized on-chain electronic interaction, thus promoting the bulk charge transport. Furthermore, the donor blocks of the donor-acceptor copolymers have been structurally varied to evaluate their influence on its electronic properties. Additionally, and since the BAI acceptor units does not carry solubilizing side chains, the donor blocks have also been used to induce a sufficient solubility.

\section{Experimental section}

\section{Materials}

Reagents were purchased from Sigma-Aldrich, Fischer Scientific or TCI and used without further purification, unless otherwise indicated. For the photophysical studies, the solutions were prepared with solvents of spectroscopic grade or equivalent: 2-methyltetrahydrofuran (2-MeTHF, anhydrous/inhibitor free, Sigma-Aldrich), toluene (tol, 99+\% for spectroscopy, Acros Organics) and analytical grade chloroform (Fischer Chemical). Silver nitrate $\left(\mathrm{AgNO}_{3}\right.$, for analysis, Panreac), Ferrocene (for synthesis, Merck) and tetrabutylammonium hexafluorophosphate $\left(\mathrm{NBu}_{4} \mathrm{PF}_{6}, \quad \geq 99.0 \%\right.$ for electrochemical analysis, Sigma-Aldrich) were used in cyclic voltammetry assays without further purification.

BAI1 was obtained from double annulation of indigo, ${ }^{13,19}$ as described in Scheme 1; 4,4-didodecyl-4H-cyclopenta[1,2-b:5, $\left.4-b^{\prime}\right]$ dithiophene $\left(\mathbf{C}_{\mathbf{1 2}} \mathbf{C P D T}\right)$ was synthesized as previously described. $^{25}$ 4,8-Bis((2-butyloctyl)oxy)benzo[1,2- $\left.b: 4,5-b^{\prime}\right]$ dithiophene (BDT) was obtained from TCI and used without further purification.

\section{Thin films preparation}

Polymer thin films were obtained with a desktop precision spin-coating system, model P6700 series from Speedline Technologies, as described elsewhere. ${ }^{26,27}$ Solutions for spin-coating were prepared by adding $2 \mathrm{mg}$ of the polymers to $200 \mu \mathrm{L}$ of chloroform solution, with stirring in the dark, at room temperature, overnight. Thin films from the samples were obtained by deposition of $c a$. $50 \mu \mathrm{L}$ from a solution of the compounds into a circular sapphire substrate $(10 \mathrm{~mm}$ diameter $)$ followed by spin-coating $(2500 \mathrm{rpm})$ in a nitrogen-saturated atmosphere (2 psi).

\section{Photophysical and electrochemical studies}

Absorption and fluorescence spectra were recorded on Agilent Cary 5000 UV-Vis-NIR and Horiba-Jobin-Ivon SPEX Fluorog 3-22 spectrometers respectively. Absorption spectra of the transparent thin films were obtained in absorption mode using a clean sapphire substrate as the reference sample. The fluorescence spectra were corrected for the wavelength response of the system. For the steady state and time resolved emission experiments, the absorption at the excitation wavelength was kept below 0.1 values to avoid aggregation effects. Acquisition of the photoluminescence spectra of the polymers at NIR were performed with Hamamatsu R5509-42 photomultiplier, cooled to $193 \mathrm{~K}$ in a liquid nitrogen chamber (Products for Research model PC176TSCE-005), connected to the fluorimeter. The colour parameters were determined according to the CIE (Commission Internationale de l'Eclairage proceedings) 1931 scale diagram. ${ }^{28}$ The $x$ and $y$ colour parameters were determined with the acquisition of the transmittance spectra using Shimadzu UV-2600 equipped with Colour Analysis software.

Fluorescence quantum yields $\left(\varphi_{\mathrm{F}}\right)$ of the polymers in solution were determined with three replicates, by William's method, ${ }^{29}$ using indocyanine green (IR-125 in ethanol, $\left.\varphi_{\mathrm{F}}=0.132\right)^{30}$ as the standard. The $\varphi_{\mathrm{F}}$ of the model monomers in solution were measured using the absolute method with a Hamamatsu Quantaurus QY absolute photoluminescence quantum yield spectrometer, model C11347 (integration sphere). The $\varphi_{\mathrm{F}}$ of the polymers in thin films were measured by the absolute method with an integrating sphere module, as described elsewhere. ${ }^{26,31,32}$ The following equation was used to determine the $\varphi_{\mathrm{F}}$ of the BAI polymers thin films:

$$
\varphi_{\mathrm{F}}(\text { solid })=\frac{\int^{\text {Sample }} I(\lambda) \mathrm{d} \lambda}{\left(\int^{\text {scatter_saffire }} I(\lambda) \mathrm{d} \lambda-\int^{\text {scatter_sample }} I(\lambda) \mathrm{d} \lambda\right)}
$$

where $\varphi_{\mathrm{F}}$ (solid) is the fluorescence quantum yield of the polymer thin film, $\int{ }^{\text {Sample }} I(\lambda) \mathrm{d} \lambda$ is the integrated area under the emission of the polymer thin film (which excludes the integration of the Rayleigh peak), $\int^{\text {scatter_saffire }} I(\lambda) \mathrm{d} \lambda$ is the integrated area under the Rayleigh peak of a sample containing only a clean sapphire support, and $\int^{\text {scatter_sample }} I(\lambda) \mathrm{d} \lambda$ is the integrated area under the Rayleigh peak in the emission spectra of the polymer in the thin film.

Fluorescence decays were measured using a home-built picosecond time-correlated single photon counting (ps-TCSPC) apparatus described elsewhere. ${ }^{33}$ Excitation was performed with the second harmonic, $372 \mathrm{~nm}$ or $402 \mathrm{~nm}$ (generated with a Spectra Physics GWU-23PS module) from a picosecond Spectra Physics mode-lock Tsunami laser (Ti:Sapphire) model 3950 (80 $\mathrm{MHz}$ repetition rate, tuning range 700-1000 nm), pumped by a Millennia Pro-10s, continuous wave, solid-state laser $(532 \mathrm{~nm})$. The fluorescence decays and the instrumental response function (IRF) were collected using 1024 channels in a time scale up to 24.4 ps per channel (using a Spectra Physics frequency divider, Pulse picker model 3980-2s, to reduce the fundamental laser repetition rate). Deconvolution of the fluorescence decay curves was performed using a modulation function method, as implemented by G. Striker in the SAND program, and previously reported in the literature. ${ }^{34}$ 
The time resolved ultrafast transient absorption measurements were collected in a broadband HELIOS spectrometer (350-1600 nm) from Ultrafast Systems as elsewhere described. ${ }^{35}$ The transient absorption data was obtained with excitation at $420 \mathrm{~nm}$ and $770 \mathrm{~nm}$ and probed in the 350-800 $\mathrm{nm}$ and 800-1600 $\mathrm{nm}$ range. The measurements in solution were obtained in a $2 \mathrm{~mm}$ quartz cuvette, with optical density $\approx 0.1-0.3$ at the pump excitation wavelength. To avoid photodegradation low laser pump energies were used $(\leq 100 \mathrm{~nJ})$ at the excitation wavelengths $420 \mathrm{~nm}$ and $770 \mathrm{~nm}$ and the solutions were stirred during the experiments or kept in movement using a motorized translating sample holder. The spectral chirp of the data was corrected using Surface Xplorer PRO program from Ultrafast Systems. Global analysis of the data (using a sequential model) was performed using Glotaran software. ${ }^{36}$ Cyclic voltammetry experiments were carried out using an Autolab potentiostat/galvanostat PGSTAT204 running with NOVA 2.1 software and a three-electrode system in a onecompartment electrochemical cell of capacity $5 \mathrm{~mL}$. A glassy carbon electrode (GCE) ( $d=3 \mathrm{~mm}$ ) was the working electrode, glassy carbon (GC) $(d=1.6 \mathrm{~mm})$ wire was the counter electrode and $\mathrm{Ag} / \mathrm{Ag}^{+}$(0.01 $\mathrm{M}$ silver nitrate, $\mathrm{AgNO}_{3}$, in $0.1 \mathrm{M}$ tetrabutylammonium hexafluorophosphate, $\mathrm{NBu}_{4} \mathrm{PF}_{6}$, in $\mathrm{MeCN}$ ) was the reference electrode. Ferrocene/ferrocenium $\left(\mathrm{Fc} / \mathrm{FC}^{+}\right)$redox couple were used as the internal reference. The reference and polymers were dissolved in $0.1 \mathrm{M} \mathrm{NBu}_{4} \mathrm{PF}_{6}$ in $\mathrm{CHCl}_{3}$ solution to acquire cyclic voltammograms with a $50 \mathrm{mV} \mathrm{s}{ }^{-1}$ scan rate with a window potential between $-1.6 \mathrm{~V}$ to $1.0 \mathrm{~V}\left(v s . \mathrm{Ag} / \mathrm{Ag}^{+}\right)$.

\section{Results and discussion}

\section{Synthesis and characterization}

The general scheme for the synthesis of the monomeric BAI precursors BAI2 and BAI4 is depicted in Scheme 2. For the synthesis of 7,14-di(thiophen-2-yl)diindolo[3,2,1-de: $3^{\prime}, 2^{\prime}$, $\left.1^{\prime}-i j\right][1,5]$ naphthyridine-6,13-dione (BAI1) indigo is first subjected to a double annulation reaction to form the BAI core. Hereby, a twofold amidation followed by an intramolecular aldol condensation takes place. ${ }^{6,13,37}$ In the second step, the 2-positions of the thiophene rings are brominated to form 7 ,

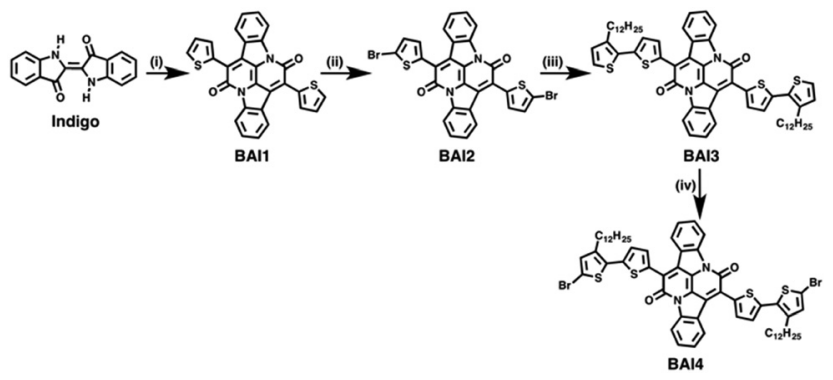

Scheme 2 Synthesis of BAI monomers. Reaction conditions: (i) 2-thienylacetyl chloride, xylene, $145^{\circ} \mathrm{C}, 48$ h, 38.5\%; (ii) NBS, chloroform, rt, 15 h, 69.7\%; (iii) 2-(trimethylstannyl)-3-dodecylthiophene, $\mathrm{Pd}\left(\mathrm{PPh}_{3}\right)_{4}$, $\mathrm{P}\left(\mathrm{o}\right.$-tol) 3 , toluene, $100{ }^{\circ} \mathrm{C}, 4$ days, $1.5 \%$; (iv) NBS, chloroform, rt, 12 h, $87.1 \%$. 14-bis(5-bromothiophen-2-yl)diindolo[3,2,1-de:3', $\left.2^{\prime}, 1^{\prime}-i j\right][1,5]$ napht-hyridine-6,13-dione (BAI2). A subsequent Stille-type coupling reaction, catalyzed by tetrakis(triphenylphosphane) palladium(0) and tri-o-tolylphosphine as a ligand, ${ }^{13}$ of BAI2 and 2-(trimethylstannyl)-3-dodecylthiophene is used to extend the thiophene substituent into a bithiophene. 7,14-Bis( $3^{\prime}$ dodecyl-[2,2'-bithiophene]-5-yl)diindolo[3,2,1-de:3', $\left.2^{\prime}, 1^{\prime}-i j\right][1,5]$ naphthyridine-6,13-dione (BAI3) is finally brominated in the absence of light into 7,14-bis( $5^{\prime}$-bromo-3'-dodecyl-[2,2' bithiophene]-5-yl)diindolo[3,2,1-de: $\left.3^{\prime}, 2^{\prime}, 1^{\prime}-i j\right][1,5]$ naphthyridine-6,13-dione (BAI4).

The Stille-type cross-coupling reaction of the two different BAI monomers (BAI2 and BAI4) with distannylated CPDT (4,4bis(2-octyldodecyl)-2,6-bis(trimethylstannyl)-4H-cyclopenta[1, $\left.2-b: 5,4-b^{\prime}\right]$ dithiophene-2,6-diyl, C9) was carried out as previously described for the synthesis of corresponding indigo-containing copolymers. ${ }^{38}$ Hereby, tetrakis(triphenylphosphane)palladium(0), $\mathrm{Pd}\left(\mathrm{PPh}_{3}\right)_{4}$, was used as a catalyst in a microwave-assisted coupling reaction. After irradiation at $125{ }^{\circ} \mathrm{C}$ for 25 minutes, the reaction mixture was stirred at $90{ }^{\circ} \mathrm{C}$ for a further $72 \mathrm{~h}$ (Scheme 3). The copolymers PBAIC-1 and PBAIC-2 were precipitated into cold methanol and fractionated by Soxhlet extraction. For the synthesis of the BAI-benzodithiophene copolymer PBAIBD-1, the reaction conditions were modified according to a report by He et al. ${ }^{13}$ Under inert conditions, tris(dibenzylideneacetone)dipalladium(0) as the catalyst and tri(o-tolylphosphane) as the ligand together with the monomers BD5 and BAI2 were dissolved in toluene and heated to $100{ }^{\circ} \mathrm{C}$. After six days, the copolymer was precipitated and Soxhlet extracted (Scheme 3).

PBAIC-1 and PBAIBD-1 show moderate molecular weights $\overline{M_{\mathrm{n}}}$ of $8-11 \mathrm{~kg} \mathrm{~mol}{ }^{-1}$, with $\mathrm{PD}=1.33$ and 1.39 , respectively, resulting possibly from a limited solubility of the products. The two additional dodecylthiophene moieties in copolymer PBAIC-2 increase the solubility of the polymer, thus probably causing its higher molecular weight and polydispersity $\left(\overline{M_{\mathrm{n}}}=11-23 \mathrm{~kg} \mathrm{~mol}^{-1}\right.$, $\mathrm{PD}=1.94)$. Nevertheless, with a value below 2 , the PDI index is still

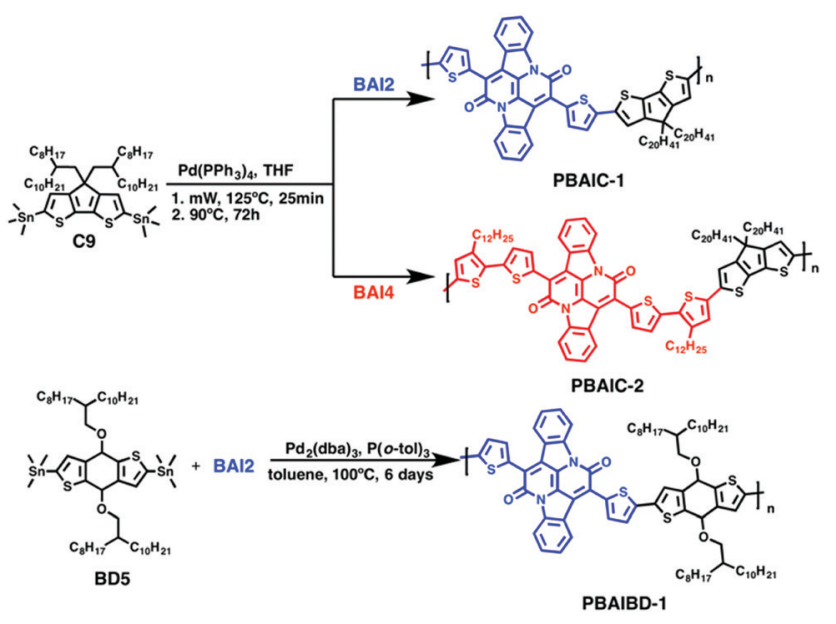

Scheme 3 General synthetic scheme for the generation of the BAI-based copolymers: PBAIC-1, PBAIC-2 and PBAIBD-1. 
as expected for polycondensations. For further synthetical procedures and structural characterization, please consult the ESI. $\dagger$

\section{Optical and electrochemical properties}

The photophysical properties of the three BAI copolymers were investigated in solution (2-MeTHF and toluene) and in thin films and compared to their monomeric units. In Fig. 1, the absorption and emission spectra of PBAIC-1 and PBAIC-2 in 2-MeTHF solution are compared with BAI1 and $\mathbf{C}_{\mathbf{1 2}}$ CPDT, PBAIBD-1 is compared to BAI1 and BDT. The change of the phenyl groups of Cibalackrot to the electron-richer thiophene groups in BAI1 lead to a bathochromic shift of the absorption maximum of $\sim 40 \mathrm{~nm}: 547 \mathrm{~nm}$ for Cibalackrot to $588 \mathrm{~nm}$ for BAI1 (Fig. SI4 and Table SI2, ESI $\dagger$ ). Generally, incorporation of the BAI chromophores into the copolymers causes a significant red shift of the absorption and emission of these in comparison to BAI1.

The shape of the absorption spectra is found similar for the three copolymers, with two broad absorption bands: one (I) in the 300-500 $\mathrm{nm}$ region and another (II), broader, at 550-1000 nm. The bathochromic shift of band I of the copolymers (PBAIC-1, PBAIC-2 and PBAIBD-1) that may be assigned to the donor units, in comparison to the absorption bands of the monomeric donor chromophores (BDT and CPDT), may indicate the conjugative interaction between the subunits. The interplay of first and second absorption band of the copolymers causes a green color of the copolymers, in contrast to the purple color characteristic of BAI1 (Fig. 1). The red shift of the second (II) absorption band in comparison with BAI1 can be associated to an extended $\pi$-conjugation and a strong donor-acceptor character of the copolymers. PBAIC-1 and PBAIC-2 (with the CPDT moiety as the electron donor unit) in 2-MeTHF solution display emission spectra with bands in the 800-1200 nm region, with peak maxima at 997 and $982 \mathrm{~nm}$, respectively, while PBAIBD-1 shows an emission spectrum with two peaks: $778 \mathrm{~nm}$ (visible region) and $944 \mathrm{~nm}$ (NIR), see Table 1. The electronic spectra of the three copolymers in toluene (Fig. SI5, $\mathrm{ESI} \dagger)$ are almost identical to the spectra in 2-MeTHF solutions

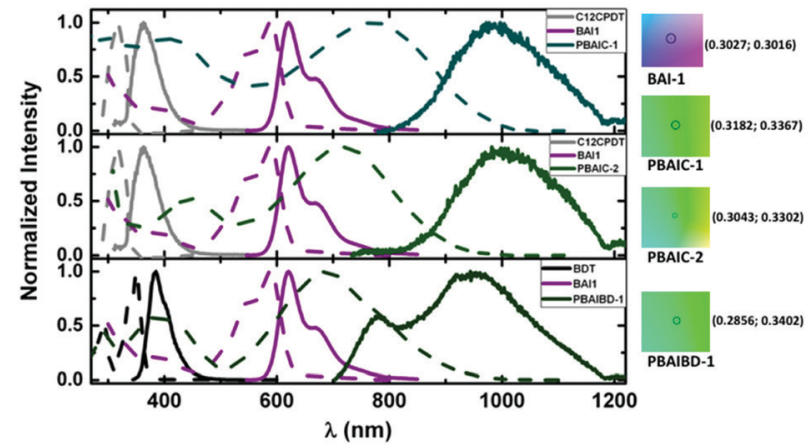

Fig. 1 Normalized absorption (dashed lines) and emission spectra (solid lines) of BAI copolymers together with respective model chromophores in 2-MeTHF at $298 \mathrm{~K}$. On the right side, the color parameters in the CIE 1931 chromaticity scale ${ }^{28}$ of the absorption spectra of BAI-1 and BAI copolymers in 2-MeTHF. (see Table SI3, ESI $\dagger$ ). The introduction of the donor CPDT unit (from PBAIC-1), instead of BDT (PBAIBD-1), leads to a red-shift of the absorption either in solution, or in solid state. Nonetheless, solid state absorption bands are red-shifted relative to the solution spectra (Fig. SI5, ESI $\dagger$ ). Fluorescence quantum yields $\left(\varphi_{\mathrm{F}}\right)$ of the BAI-containing copolymers obtained in 2-MeTHF, at $298 \mathrm{~K}$ and $77 \mathrm{~K}$, and in thin films at $298 \mathrm{~K}$ (Table 1) are roughly similar to the solid state (thin films) values. The spatial structures of PBAIC-1, PBAIC-2 and PBAIBD1 as donor-acceptor copolymers seem to preclude aggregation caused quenching (ACQ) effects. Moreover, the $\varphi_{\mathrm{F}}$ values of the BAI copolymers at $77 \mathrm{~K}$ are about four times higher than in solution at $293 \mathrm{~K}$ (see Fig. SI6 and Table SI4, ESI $\dagger$ ), as a factor of interest for potential applications, e.g. as the photoactive layer of NIR organic photodiodes. The PL emission area of the three polymers presented a linear course with the increase of the absorption at the excitation wavelength, in solution (Fig. SI7, $\mathrm{ESI} \dagger$ ), ruling out aggregation effects of the polymer in 2-meTHF.

The electrochemical properties and energy levels of BAI copolymers were determined in chloroform solution (Table 2). The characteristic, quasi-reversible double reduction of the amide groups, correlated to the BAI moiety, is prominent in the cyclic voltammogram of PBAIBD-1. Moreover, the oxidation and reduction potentials are similar to those found in the literature to D-A type polymers based on bay-annulated indigo and thiophene units. ${ }^{13,16,19}$

The energies of the HOMO and LUMO levels were obtained according to eqn (2) and (3). ${ }^{39}$

$$
\begin{gathered}
E_{\mathrm{HOMO}}=-\left(E_{\mathrm{Ox}}^{\mathrm{onset}}+4.8\right)-E_{\mathrm{FOC}} \\
E_{\mathrm{LUMO}}=E_{\mathrm{HOMO}}+E_{0-0}
\end{gathered}
$$

The energy of the HOMO, $\mathrm{E}_{\mathrm{HOMO}}$, was calculated from the onset oxidation potential, $E_{\mathrm{ox}}^{\text {onset }}$, plus 4.8 , which is the difference between the reference energy level of ferrocene below the vacuum level and the oxidation potential of ferrocene, $E_{\mathrm{FOC}}$, given by the potential of $\mathrm{FOC} / \mathrm{FOC}^{+} v s . \mathrm{Ag} / \mathrm{Ag}^{+}$measured by cyclic voltammetry in solution (Fig. 2). The LUMO energy, $E_{\text {LUMO }}$, was obtained from the optical energy gap $E_{0-0}$, calculated from the interception of the normalized UV-Vis absorption and fluorescence spectra, through eqn (3).

The HOMO-levels of the CPDT-based polymers are similar with values of $-4.96 \mathrm{eV}$ (PBAIC-1) and -4.95 eV (PBAIC-2), respectively, and correlate well with values determined from the onset of absorption (Table SI5, ESI $\dagger$ ). The LUMO-levels, about -3.5 to $-3.9 \mathrm{eV}$, is closely correlated to the $E_{\mathrm{LUMO}}=-3.6 \mathrm{eV}$, previously attributed to the model compound BAI1. ${ }^{40}$ CPDTbased polymers also presented smaller band gaps than PBAIBD-1 (built with BDT as donor unit), thus, CPDT may be a stronger electron donor unit than BDT and, therefore, lead to higher HOMO energy levels (Table 2).

The band gap energies of BAI copolymers vary between 1.3-1.5 eV, when determined either by CV, or UV-spectroscopy (Table 2). Narrow band gap $\left(E_{\mathrm{g}}\right)$ conjugated polymers, $1.1 \leq E_{\mathrm{g}}$ $\leq 2.1 \mathrm{eV}$, have especially low lying LUMO energies making 
Table 1 Room temperature spectroscopic data (absorption and fluorescence emission maxima together with Stokes shift, $\Delta_{\mathrm{SS}}$ ) and fluorescence quantum yield $\left(\varphi_{\mathrm{F}}\right)$ for the BAl copolymers and model monomers at $T=298 \mathrm{~K}$

\begin{tabular}{|c|c|c|c|c|c|}
\hline Compound & Medium & $\lambda^{\mathrm{abs}}(\mathrm{nm})$ & $\lambda^{\mathrm{em}}(\mathrm{nm})$ & $\Delta_{\mathrm{ss}}\left(\mathrm{cm}^{-1}\right)$ & $\varphi_{\mathrm{F}}$ \\
\hline $\mathrm{C}_{12} \mathrm{CPDT}$ & 2-meTHF & 316 & 363 & 4097 & $0.048^{a}$ \\
\hline BDT & & 351 & 385 & 2516 & 0.089 \\
\hline BAI-1 & & $550(\mathrm{sh})^{b}, 588$ & $621,669(\mathrm{sh})^{b}$ & 904 & 0.389 \\
\hline \multirow[t]{2}{*}{ PBAIC-1 } & 2-MeTHF & 404,775 & 997 & 2873 & $0.015 \pm 0.002$ \\
\hline & Film & 392,821 & 1074 & 2869 & $0.016 \pm 0.001$ \\
\hline \multirow[t]{2}{*}{ PBAIC-2 } & 2-MeTHF & 447,716 & 982 & 3783 & $0.013 \pm 0.002$ \\
\hline & Film & 457,753 & 1060 & 3846 & $0.011 \pm 0.001$ \\
\hline \multirow[t]{2}{*}{ PBAIBD-1 } & 2-MeTHF & 378,688 & 944 & 3942 & $0.018 \pm 0.002$ \\
\hline & Film & 429,749 & 1118 & 4407 & $0.0066 \pm 0.0001$ \\
\hline
\end{tabular}

${ }^{a} \varphi_{\mathrm{F}}=0.05$ in methylcyclohexane in ref. $25 .{ }^{b} \mathrm{sh}=$ shoulder in the spectra; see the text for more details.

Table 2 Experimental electrochemical parameters and energy levels ${ }^{a}$ of BAl copolymers determined in solution

\begin{tabular}{llllllll}
\hline Polymer & $\begin{array}{l}E_{\mathrm{ox}}^{\text {onst }} \\
(\mathrm{V})\end{array}$ & $\begin{array}{l}E_{\mathrm{HOMO}} \\
(\mathrm{eV})\end{array}$ & $\begin{array}{l}E_{\mathrm{LUMO}} \\
(\mathrm{eV})\end{array}$ & $\begin{array}{l}E_{\mathrm{g}} \\
(\mathrm{eV})\end{array}$ & $\begin{array}{l}E_{\mathrm{g}}{ }^{b} \\
(\mathrm{eV})\end{array}$ & $\begin{array}{l}E_{1 / 2}^{\mathrm{Ox}} \\
(\mathrm{V})\end{array}$ & $\begin{array}{l}E_{1 / 2}^{\mathrm{Red}} \\
(\mathrm{V})\end{array}$ \\
\hline PBAIC-1 & 0.0929 & -4.96 & -3.57 & 1.39 & 1.48 & 0.27 & -1.15 \\
PBAIC-2 & 0.0909 & -4.95 & -3.54 & 1.42 & 1.29 & 0.23 & -1.18 \\
PBAIBD-1 & 0.514 & -5.38 & -3.85 & 1.52 & 1.54 & 0.25 & -1.12
\end{tabular}

${ }^{a} E_{\mathrm{FOC}}=0.064 \mathrm{~V}$ determined in $0.1 \mathrm{M} \mathrm{NBu}_{4} \mathrm{PF}_{6}$ in $\mathrm{CHCl}_{3} .{ }^{b}$ Estimated from the onset of absorption. See eqn (3) and (4) and Table SI5 (ESI) for further details.

PBAIC-1

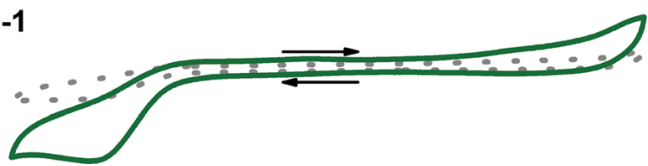

PBAIC-2
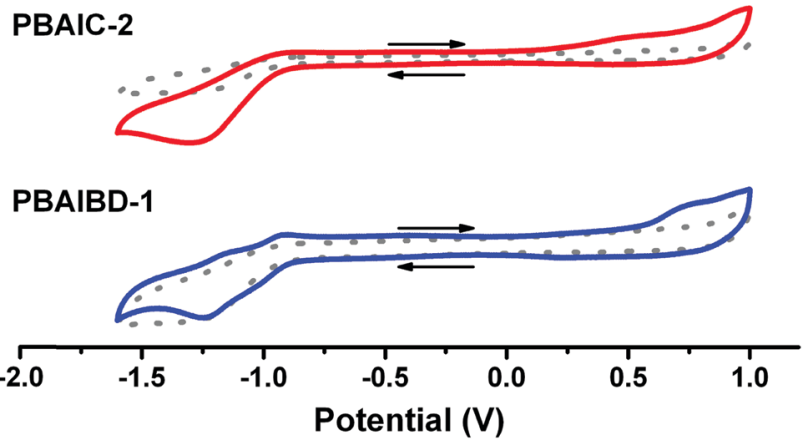

Fig. 2 Cyclic voltammograms of the BAl copolymers in $\mathrm{CHCl}_{3}$ solution obtained with a scan rate of $100 \mathrm{mV} \mathrm{s}^{-1}$. Dotted lines represent the blank voltammogram, acquired with the electrolyte solution $\left(0.1 \mathrm{M} \mathrm{NBu}_{4} \mathrm{PF}_{6}\right.$ in $\left.\mathrm{CHCl}_{3}\right)$.

them excellent candidates for n-type OFET materials. ${ }^{41}$ Moreover, these BAI copolymers also display optical absorption in the near-IR region of the electromagnetic spectrum, ideal for OPV devices. ${ }^{21}$ Also, because these polymers contain both donor and acceptor moieties on their backbones, they are promising candidates for single-component polymer solar cells by replacing polymer blends used as active layers in manufacturing all-polymer solar cells, and thus, overcome drawbacks such as miscibility between the donor and acceptor polymer components and gradual phase separation of polymer blend films. ${ }^{42-44}$

\section{Time-resolved spectroscopy studies}

Femtosecond transient absorption (fs-TA) was further used to characterize the excited state dynamics of the copolymers and two model monomers (BAI1 and BDT) in solution and in thin films (Fig. SI8-SI11 and Table SI6, ESI $\dagger$ ). From Fig. 3, it is seen that the fs-TA spectra of the copolymers are composed of a broad positive excited state absorption (ESA) in the 880-1600 nm range, and negative TA bands between $350 \mathrm{~nm}$ and $955 \mathrm{~nm}$ matching with those in Fig. 1 attributed to groundstate absorption (GSA).

In solution after about $\sim 5$ ps (PBAIC-2) and $\sim 10$ ps (PBAIBD-1) the initially formed ESA band blue-shifts by $\sim 20 \mathrm{~nm}$ and thereon the band maxima remain unchanged. Since there are no additional bands at longer decay times (that could be attributed, e.g., to triplet excited state formation in the polymers) the positive TA bands are attributed to the $\mathrm{S}_{1} \rightarrow \mathrm{S}_{n}$ ESA. This behavior contrasts with that of the monomeric BAI1 chromophore where in addition to the $\mathrm{S}_{1} \rightarrow \mathrm{S}_{n}$ ESA (in the

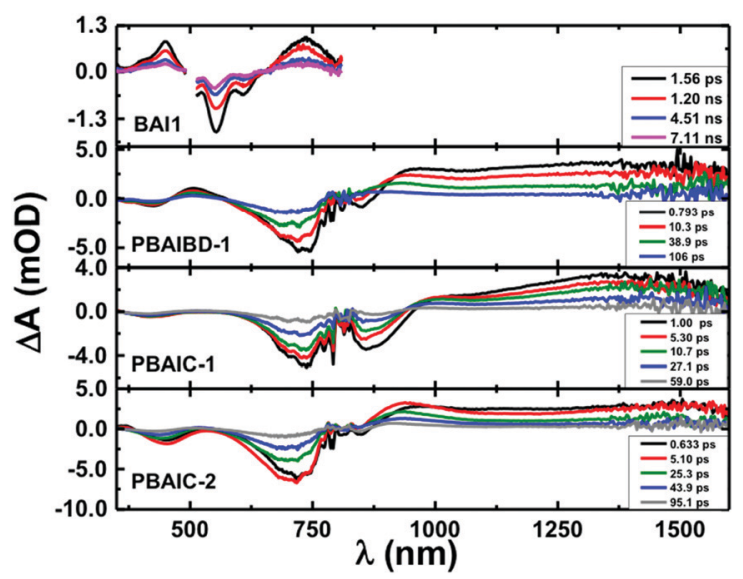

Fig. 3 Room temperature time resolved transient absorption spectra (fsTA) for the BAI copolymers and monomeric BAl1 in aerated 2Me-THF solution collected with excitation at $770 \mathrm{~nm}$ and $500 \mathrm{~nm}$, respectively. The data was collected in the 350-825 nm and 800-1600 nm ranges and merged together using Surface Xplorer software with normalization of the two surfaces in the $800-825 \mathrm{~nm}$ range. This explains why the spectral data in the 770-830 $\mathrm{nm}$ region shows some noise. 
350-480 $\mathrm{nm}$ and 665-800 $\mathrm{nm}$ ), GSA, and stimulated emission in the (510-630 nm) bands, a triplet-triplet absorption band is also observed with a triplet lifetime of $\tau_{\mathrm{T}}=54 \mu \mathrm{s}$ (presented in the ns-TA absorption spectra, Fig. SI9, ESI $\dagger$ ). For PBAIBD-1 a characteristic ESA band is seen in the 460-560 range, as also found for the monomeric BDT unit. However, contrary to monomeric BDT, in PBAIBD-1 this band disappears after 106 ps (see Fig. SI10, SI11, ESI, $\dagger$ and Fig. 2). For monomeric BDT the $\mathrm{S}_{1} \rightarrow \mathrm{S}_{n}$ ESA band was found to be strongly overlapped with the $\mathrm{T}_{1} \rightarrow \mathrm{T}_{n}$ ESA band, as shown by comparison with the triplet absorption spectra obtained by ns-TA, $\left(\tau_{\mathrm{T}}=99 \mu \mathrm{s}\right)$, Fig. SI10 (ESI $\dagger$ ). The data support (i) the pronounced conjugation between BAI1 and BDT moieties in the PBAIBD-1 copolymer, which promotes electron-delocalization, as shown by the significant red-shift of the absorption band when compared with the monomeric BAI1 (Fig. 1), and (ii) the absence of triplet state formation in the polymers, due to the fast deactivation of the excited state. The mechanism behind this will be discussed below.

Global analysis of representative kinetic traces of the copolymers shows that transient decays are well fitted with the sum of three exponentials. The best-fit results and pre-exponential values are presented in Table 3 (also in Table SI6 and Fig. SI11 in the ESI $\dagger$ ). Either in solution, or in thin films, the copolymers show a first decay transient with values in the 0.71-8.9 ps range, a second one with values in the 14.9-58.4 ps range and a longer decay transient with values ranging from 55.6 ps to 1948 ps. BAI1 transient lifetimes of 66 ps, 5201 ps and $54 \mu$ s were determined. The latter was fixed in the analysis to the triplet lifetime obtained by ns-TA (Fig. SI9, ESI $\dagger$ ). The two shortest decay times are in agreement with the double-exponential decay behavior obtained from the fluorescence decays of BAI1 (fluorescence lifetimes, $\tau_{\mathrm{F}}=140 \mathrm{ps}$ and $4680 \mathrm{ps}$, Fig. SI12, ESI $\dagger$ ). BDT transient decays are well fitted with a double exponential decay law, with transient lifetimes of 313 ps (also in agreement with $\tau_{\mathrm{F}}=560$ ps obtained by TCSPC, Fig. SI13, ESI $\dagger$ ) and $99 \mu \mathrm{s}$, that was fixed in the analysis to the triplet lifetime obtained by ns-TA (Fig. SI14 in ESI $\dagger$ ).

Table 3 Pre-exponential values $\left(a_{i}\right)$ resulting from the global analysis to the representative kinetics of the time-resolved transient absorption data, together with the rate constants for energy transfer processes $\left(k_{\mathrm{ET}}\right)$ for the investigated BAI copolymers in 2MeTHF solution

\begin{tabular}{llccccc}
\hline Polymer & $\lambda(\mathrm{nm})$ & & $a_{i}$ & & $k_{\mathrm{ET}}(1)\left(\mathrm{s}^{-1}\right)$ & $k_{\mathrm{ET}}(2)\left(\mathrm{s}^{-1}\right)$ \\
\hline PBAIC-1 & $\tau_{i}(\mathrm{ps})$ & 0.71 & 19.4 & 114.7 & & \\
& 911 & -0.494 & -0.506 & 0.059 & $\mathrm{NA}^{a}$ & $4.55 \times 10^{10}$ \\
& 1200 & 0.194 & 0.645 & 0.160 & & \\
PBAIC-2 & $\tau_{i}(\mathrm{ps})$ & 2.4 & 36.7 & 230.8 & & \\
& 911 & -0.560 & 0.895 & 0.105 & $4.11 \times 10^{11}$ & $2.12 \times 10^{10}$ \\
& 1200 & 0.232 & 0.746 & 0.022 & & \\
PBAIBD-1 & $\tau_{i}(\mathrm{ps})$ & 8.9 & 49.7 & 163.1 & & \\
& 505 & 0.506 & 0.196 & 0.298 & & \\
& 911 & 0.440 & -0.271 & 0.560 & $1.11 \times 10^{11}$ & $1.83 \times 10^{10}$ \\
& 1200 & 0.292 & 0.848 & 0.642 & &
\end{tabular}

${ }^{a} \mathrm{NA}=$ not applicable. Shorter $\tau_{1}(<10 \mathrm{ps})$ component attributed to solvation dynamics.
The shortest decay time component $\left(\tau_{1}\right)$ which, at shorter emission wavelengths is associated to a negative preexponential for the PBAIC-1 copolymer (Table 3), should be considered to be associated to solvent dynamics (in THF with characteristic times ranging from $0.43 \mathrm{ps}$ to $0.94 \mathrm{ps}),{ }^{45}$ or attributed to vibrational relaxation due to the depopulation of a hot vibrational state generated through the $770 \mathrm{~nm}$ excitation (since excitation was not performed in the lowest energy 0-0 electronic transition). ${ }^{38}$ Yet, this is not the case with PBAIC-2 and PBAIBD-1, where a longer component is observed with 2.4 ps and 8.9 ps (Table 3), respectively, in addition to a longer $\tau_{3}$ component in the order of hundreds of ps. These $\tau_{1}$ and $\tau_{2}$ decay components are associated to excitation energy migration within different segments of the polymer chain (higher to lower energy conjugated segments) ending up in the $\tau_{3}$ decay component which mirrors the decay of the more relaxed segment to the ground state.

From the donor-acceptor (D-A) character of the copolymers, one can determine the energy transfer $\left(k_{\mathrm{ET}}\right)$ rate constant from: ${ }^{38,46-48}$

$$
k_{\mathrm{ET}}=\frac{1}{\tau_{1,2}}-\frac{1}{\tau_{0}}
$$

where $\tau_{0}$ stands for the donor lifetime in the absence of acceptor and $\tau_{1,2}$ for the decay time of the donor in the polymer (with acceptor units). Due to the chemical structure of the corresponding repeat units (Scheme 1), the monomeric $\mathbf{C}_{12}$ CPDT or BDT are taken as model donor units of the copolymers. Therefore for PBAIC-1,2 $\tau_{0}=166 \mathrm{ps}^{25}$ (related to $\mathbf{C}_{12}$ CPDT) and for PBAIBD-1 $\tau_{0}=560$ ps (related to BDT, Fig. S11, ESI $\dagger$ ) were used in eqn (4). The two decay components $\left(\tau_{1}\right.$ and $\tau_{2}$ ) describe contributions of the energy donors in the presence of energy acceptors. ${ }^{49}$ This three exponential fit of the transient decays basically mirrors the fact that energy transfer (ET) processes lead to nonexponential decays and that the two shortest decay times, and respective pre-exponential factors, characterize, as a whole, the ET process. Indeed, from the preexponential factors in solution (Table 3), except for the longer component (associated to a relaxed exciton segment decaying to the ground state) and the shorter component of PAIBC-1 (associate to solvent dynamics) the ET process can be fully characterized by the two mentioned components. Considering the simplest case of Coulombic (Förster) energy transfer, in which the energy donor units are distributed in a medium with a uniform concentration of acceptors, $C_{\mathrm{A}}$, the fluorescence decay function of the donor, $I_{\mathrm{D}}(\mathrm{t})$, in the presence of acceptors can be obtained by eqn (5), ${ }^{49,50}$

$$
I_{\mathrm{D}}(t)=\exp \left(-t / \tau_{0}\right) \exp \left[-(4 / 3) \pi^{3 / 2} R_{0}{ }^{3} C_{\mathrm{A}}\left(t / \tau_{0}\right)^{1 / 2}\right]
$$

with $R_{0}$ the Förster radius and $\tau_{0}$ the lifetime of the donor in the absence of an acceptor.

Although energy transfer leads to nonexponential decays, the $I_{\mathrm{D}}(t)$ for PBAIC-2 and PBAIBD-1 can be fitted with a sum of two exponentials terms (Fig. 4), which corresponded to the two shortest decay times in these copolymers (Table 3). 


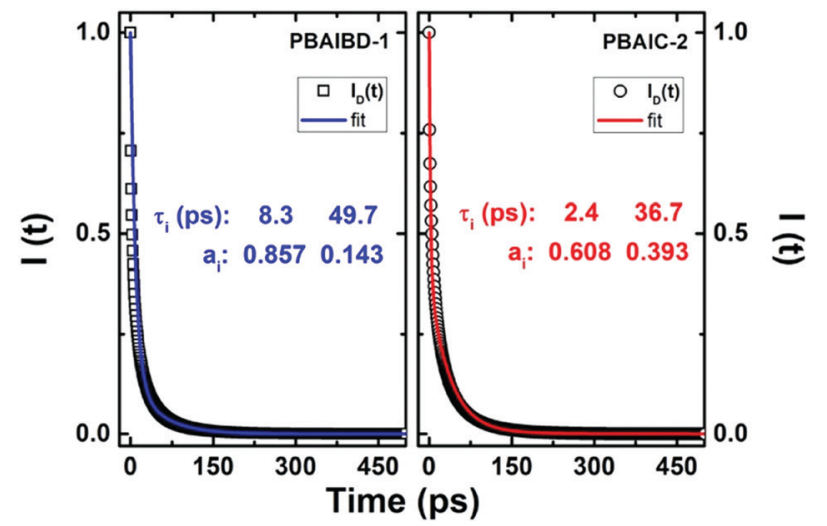

Fig. 4 Plot of the fluorescence decay function of the donor, $I_{D}(t)$, in the presence of acceptors (open circles and squares) according to eqn (5) and respective double exponential fit, $I(t)=a_{1} \exp \left(-t / \tau_{1}\right)+a_{2} \exp \left(-t / \tau_{2}\right)$, blue and red lines for the PBAIBD-1 and PBAIC-2 copolymers.

For PBAIC-2, $\tau_{2}$ is dominant particularly at longer wavelengths (representing $\sim 91 \%$ of the total decay) and should be therefore seen as governing the ET process (i.e., the ET process involving more distant D-A pairs); for PBAIBD-1 similar contributions were found for the two decay times, 50\%, indicating that short and distant $\mathrm{D}-\mathrm{A}$ pairs contribute equally to the onchain hopping decay mechanism. Additionally, the rate constants for the ET process varies from $1.83 \times 10^{10}$ to $4.11 \times 10^{11} \mathrm{~s}^{-1}$ (Table 3), characteristic of conjugated organic polymers. ${ }^{38,49}$

\section{Conclusions}

In this work, three new BAI-based donor-acceptor copolymers were synthesized, and their photoexcitation decay mechanisms investigated in solution and thin films. Even though the $\varphi_{\mathrm{F}}$ of these copolymers is found from 1 up to $2 \%$ in solution, the film values are basically identical to solution, thus showing that ACQ is not taking place. While in solution, the BAI-containing copolymers presented broad long wavelength absorption bands, with maxima varying between 688-775 $\mathrm{nm}$, in thin films, the red-shifted emission bands reach 900-1300 nm, indicating that these copolymers are a promising class of lowbandgap, circa 1.4-1.5 eV, copolymers with solid state absorption and emission widely shifted into the NIR region. The decays in the PBAIBD-1 and PBAIC-2 copolymers were further associated to an excitation energy transfer hopping that could be rationalized with a Förster-type mechanism with differing donor-acceptor contributions.

Our work uncovers not only the need for the development of new NIR-absorber polymers to featured applications e.g. in sensing and photovoltaics, but also reinforces the use of bayannulated indigo as a building block for the generation of push-pull type conjugated polymers.

\section{Conflicts of interest}

There are no conflicts to declare.

\section{Acknowledgements}

This work was supported by Project "Hylight" (no. 031625) 02/ SAICT/2017, PTDC/QUI-QFI/31625/2017, which is funded by the Portuguese Science Foundation and Compete Centro 2020. We acknowledge funding by Fundo Europeu de Desenvolvimento Regional (FEDER) through Programa Operacional Factores de Competitividade (COMPETE) and project ROTEIRO/0152/2013. The Coimbra Chemistry Centre is supported by the Fundação para a Ciência e a Tecnologia (FCT, UID/QUI/00313/2019-2020). The research leading to these results has received funding from Laserlab-Europe (grant agreement no. 284464, EC's Seventh Framework Programme). We also acknowledge Dr Daniela Pinheiro, for the assistance in the electrochemical studies, and Dr Sybille Allard, for GPC spectral data.

\section{Notes and references}

1 M. J. Melo, J. L. Ferreira, A. J. Parola and J. S. S. de Melo, Appl. Photochem., 2016, 499-530, DOI: 10.1007/978-3-31931671-0_13.

2 J. Pina, D. Sarmento, M. Accoto, P. L. Gentili, L. Vaccaro, A. Galvao and J. S. Seixas de Melo, J. Phys. Chem. B, 2017, 121, 2308-2318.

3 J. Sérgio Seixas de Melo, Photochemistry, 2018, 45, 68-100.

4 J. Sérgio Seixas de Melo, Photochemistry, 2020, 47, 196-216, DOI: $10.1039 / 9781788016520-00196$.

5 D. Pinheiro, M. Pineiro, A. M. Galvão and J. S. Seixas de Melo, Chem. Sci., 2021, 12, 303-313.

6 G. Engi, Zeitschrift für Angew. Chem., 1914, 27, 144-148.

7 J. Seixas de Melo, R. Rondao, H. D. Burrows, M. J. Melo, S. Navaratnam, R. Edge and G. Voss, J. Phys. Chem. A, 2006, 110, 13653-13661.

8 M. B. Smith and J. Michl, Annu. Rev. Phys. Chem., 2013, 64, 361-386.

9 J. L. Ryerson, A. Zaykov, L. E. Aguilar Suarez, R. W. A. Havenith, B. R. Stepp, P. I. Dron, J. Kaleta, A. Akdag, S. J. Teat, T. F. Magnera, J. R. Miller, Z. Havlas, R. Broer, S. Faraji, J. Michl and J. C. Johnson, J. Chem. Phys., 2019, 151, 184903.

10 A. Shukla, N. R. Wallwork, X. Li, J. Sobus, V. T. N. Mai, S. K. M. McGregor, K. Chen, R. J. Lepage, E. H. Krenske, E. G. Moore, E. B. Namdas and S. C. Lo, Adv. Opt. Mater., 2019, 1901350, DOI: 10.1002/adom.201901350.

11 E. D. Glowacki, G. Voss and N. S. Sariciftci, Adv. Mater., 2013, 25, 6783-6800.

12 T. Furuyama, D. Tamura, H. Maeda and M. Segi, Tetrahedron Lett., 2018, 59, 2913-2916.

13 B. He, A. B. Pun, D. Zherebetskyy, Y. Liu, F. Liu, L. M. Klivansky, A. M. McGough, B. A. Zhang, K. Lo, T. P. Russell, L. Wang and Y. Liu, J. Am. Chem. Soc., 2014, 136, 15093-15101.

14 M. A. Kolaczkowski, B. He and Y. Liu, Org. Lett., 2016, 18, 5224-5227. 
15 B. He, W. T. Neo, T. L. Chen, L. M. Klivansky, H. Wang, T. Tan, S. J. Teat, J. Xu and Y. Liu, ACS Sustainable Chem. Eng., 2016, 4, 2797-2805.

16 J. Zhu, T. Li, K. Shi, J. Wang, Y. Lin, G. Yu and X. Zhan, J. Polym. Sci., Part A: Polym. Chem., 2018, 56, 213-220.

17 J. Qi, W. Qiao and Z. Y. Wang, Chem. Rec., 2016, 16, 1531-1548.

18 D. Li, S. Yip, F. Li, H. Zhang, Y. Meng, X. Bu, X. Kang, C. Lan, C. Liu and J. C. Ho, Adv. Opt. Mater., 2020, 8, 2001201.

19 F. Verstraeten, S. Gielen, P. Verstappen, J. Kesters, E. Georgitzikis, J. Raymakers, D. Cheyns, P. Malinowski, M. Daenen, L. Lutsen, K. Vandewal and W. Maes, J. Mater. Chem. C, 2018, 6, 11645-11650.

20 X. Liu, Y. Lin, Y. Liao, J. Wu and Y. Zheng, J. Mater. Chem. C, 2018, 6, 3499-3513.

21 T. J. Wen, D. Wang, L. Tao, Y. Xiao, Y. D. Tao, Y. Li, X. Lu, Y. Fang, C. Z. Li, H. Chen and D. Yang, ACS Appl. Mater. Interfaces, 2020, 12, 39515-39523.

22 D. S. Leem, K. H. Lee, N. Li, B. W. Park, T. Choi, T. Ro, O. K. Kwon, Y. N. Kwon, T. N. Ng and S. Kim, Adv. Opt. Mater., 2020, 2001682, DOI: 10.1002/adom.202001682.

23 G. Yuan, H. Lv, H. Liu, H. He, Q. Sun, X. Zhang and S. wang, Dyes Pigm., 2020, 183, 108674.

24 F. Verstraeten, S. Gielen, P. Verstappen, J. Raymakers, H. Penxten, L. Lutsen, K. Vandewal and W. Maes, J. Mater. Chem. C, 2020, 8, 10098-10103.

25 J. Pina, A. Eckert, U. Scherf, A. M. Galvão and J. S. Seixas de Melo, Mater. Chem. Front., 2018, 2, 149-156.

26 J. Pina, J. Seixas de Melo, H. D. Burrows, A. Bilge, T. Farrell, M. Forster and U. Scherf, J. Phys. Chem. B, 2006, 110, 15100-15106.

27 A. C. B. Rodrigues, I. S. Geisler, P. Klein, J. Pina, F. J. H. Neuhaus, E. Dreher, C. W. Lehmann, U. Scherf and J. S. Seixas de Melo, J. Mater. Chem. C, 2020, 8, 2248-2257.

28 T. Smith and J. Guild, Trans. Opt. Soc., London, 1931, 33, 73-134.

29 A. T. R. Williams, S. A. Winfield and J. N. Miller, Analyst, 1983, 108, 1067-1071.

30 K. Rurack and M. Spieles, Anal. Chem., 2011, 83, 1232-1242.

31 J. C. de Mello, H. F. Wittmann and R. H. Friend, Adv. Mater., 1997, 9, 230-232.

32 L. O. Pålsson and A. P. Monkman, Adv. Mater., 2002, 14, 757-758.
33 J. Pina, J. Seixas de Melo, H. D. Burrows, A. L. Maçanita, F. Galbrecht, T. Bünnagel and U. Scherf, Macromolecules, 2009, 42, 1710-1719.

34 G. Striker, V. Subramaniam, C. A. M. Seidel and A. Volkmer, J. Phys. Chem. B, 1999, 103, 8612-8617.

35 J. Pina, M. J. R. P. Queiroz and J. S. Seixas de Melo, Photochem. Photobiol. Sci., 2016, 15, 1029-1038.

36 J. J. Snellenburg, S. P. Laptenok, R. Seger, K. M. Mullen and I. H. M. V. Stokkum, J. Statistical Software, 2012, 49, 1-22.

37 R. Tobler, Helv. Chim. Acta, 1945, 28, 901-911.

38 J. Pina, M. Alnady, A. Eckert, U. Scherf and J. S. Seixas de Melo, Mater. Chem. Front., 2018, 2, 281-290.

39 R. C. Pereira, A. D. R. Pontinha, M. Pineiro and J. S. Seixas de Melo, Dyes Pigm., 2019, 166, 203-210.

40 M. A. Kolaczkowski and Y. Liu, Chem. Rec., 2019, 19, 1062-1077.

41 K. J. Fallon, N. Wijeyasinghe, E. F. Manley, S. D. Dimitrov, S. A. Yousaf, R. S. Ashraf, W. Duffy, A. A. Y. Guilbert, D. M. E. Freeman, M. Al-Hashimi, J. Nelson, J. R. Durrant, L. X. Chen, I. McCulloch, T. J. Marks, T. M. Clarke, T. D. Anthopoulos and H. Bronstein, Chem. Mater., 2016, 28, 8366-8378.

42 Y. U. Kim, B. S. Ma, Y. Kim, S. H. Park, H. Kang, H. J. Yoon, M. J. Cho, T.-S. Kim, J. H. Kim and D. H. Choi, Chem. Eng. J., 2021, 415, 128952, DOI: 10.1016/j.cej.2021.128952.

43 C. G. Park, S. H. Park, Y. Kim, T. L. Nguyen, H. Y. Woo, H. Kang, H. J. Yoon, S. Park, M. J. Cho and D. H. Choi, J. Mater. Chem. A, 2019, 7, 21280-21289.

44 N. Y. Kwon, S. H. Park, H. Kang, A. V. Takaloo, A. K. Harit, H. Y. Woo, T. G. Kim, H. J. Yoon, M. J. Cho and D. H. Choi, J. Mater. Chem. A, 2020, 8, 20091-20100.

45 M. L. Horng, J. A. Gardecki, A. Papazyan and M. Maroncelli, J. Phys. Chem., 1995, 99, 17311-17337.

46 J. S. Seixas de Melo, J. Pina, F. B. Dias and A. L. Maçanita, Appl. Photochem., 2013, ch. 15, pp. 533-585, DOI: $10.1007 /$ 978-90-481-3830-2_15.

47 J. Pina, J. S. Seixas de Melo, J.-M. Koenen, S. Jung and U. Scherf, J. Phys. Chem. C, 2013, 117, 3718-3728.

48 J. Pina, J. S. Seixas de Melo, N. Koenen and U. Scherf, J. Phys. Chem. B, 2013, 117, 7370-7380.

49 B. Ferreira, P. F. da Silva, J. S. Seixas de Melo, J. Pina and A. Macanita, J. Phys. Chem. B, 2012, 116, 2347-2355.

50 A. Yekta, M. A. Winnik, J. P. S. Farinha and J. M. G. Martinho, J. Phys. Chem. A, 1997, 101, 1787-1792. 